\title{
Posterior Reversible Encephalopathy Syndrome (PRES) in the peripartum period
}

\author{
Jayati Nath \\ Correspondence: Jayati Nath, Professor, Obstetrics and Gynecology, MMIMSR, Mullana, \\ Ambala, Haryana; Email - jayati.nath@yahoo.com
}

Distributed under Creative Commons Attribution-Share Alike 4.0 International.

\begin{abstract}
Posterior Reversible Encephalopathy Syndrome (PRES) is defined as a clinico-pathological disorder characterized by a myriadof symptoms including altered mental status, headaches, blurring of vision and seizure, because of the involvement of the occipital and/or parietal lobes of the brain. Eclampsia is known to be the commonest etiological factors behind PRES. This is a review of literature guided clinic-radiological findings which aid in the early diagnosis and management of PRES and thereby reduce morbidities and complications related to the same.
\end{abstract}

Keywords: Convulsions, eclampsia, PRES, peripartum period, seizure.

Posterior Reversible Encephalopathy Syndrome, acronymed as PRES, is a clinico-neuroradiologically diagnosed condition which affects the occipital and parietal lobes of the brain, characterized by reversible changes occurring in the central nervous system evidenced by typical radiological features on MRI (Magnetic Resonance Imaging) or CT (Computed Tomography) imaging of the brain. Also called as Reversible Posterior Encephalopathy Syndrome (RPLS), this is a rapidly evolving neurological state characterized by altered mental status, confusion, headaches, visual disturbances especially blurring of vision and seizures ${ }^{1,3}$. The diagnosis of PRES is established by taking into account the clinical history of the patient, along with physical and neurological examination. Early diagnosis with prompt treatment may lead to complete resolution of the symptoms within 2 weeks. Failure to diagnose the entity at an early stage might result in adverse outcomes ranging from persistent and residual neurologic deficit and disabilities to even death ${ }^{2,4}$. Many etiological factors are implicated eg immunosuppressive medications, kidney failure, eclampsia, severe and malignant hypertension, systemic lupus erythematosis (SLE) . Hemorrhagic press and severe and malignant variants of PRES carry very poor prognosis for the patient with very high morbidity and mortality rates $2,3,5$.

This is a review of literature guided clinico neuroradiological findings of peripartum women with PRES to aid in early diagnosis and initiation of treatment so as to reduce the complications thereof.

\section{Discussion}

Historically this entity it is known by many other synonyms eg

- Posterior Reversible Encephalopathy Syndrome

- Reversible Posterior Leukoencephalopathy Syndrome (RPLS)

Received: $16^{\text {th }}$ January 2018. Accepted: $11^{\text {th }}$ June 2018 .

Nath J. Posterior Reversible Encephalopathy Syndrome (PRES) in the peripartum period. The New Indian Journal of OBGYN. 2018; 5(1): 8-12. 
- Hyperperfusion Encephalopathy

- Brain Capillary Leak Syndrome (BCLS)

- Hypertensive Encephalopathy (HE)

- Posterior Encephalopathia

- Occipito- Parietal Encephalopathy (OPE)

- Toxemic Posterior Encephalopathy (TPE)

This clinical entity was described for the very first time in 1996 by Hinchey et al ${ }^{1}$. Although PRES is most commonly seen and described in postpartum patients with history of antenatal hypertension, there are many other clinical conditions which might be the underlying etiological factors example hypertension - essential or chronic, preeclampsia, eclampsia, renal diseases eg. acute glomerulonephritis, nephritis secondary to SLE (lupus nephritis), HUS (hemolytic uremic syndrome), thrombotic thrombocytopenic purpura (TTP), renal transplant, immunosuppressive drugs eg cyclosporine A, tacrolimus, interferon alpha and corticosteroids, infections eg toxemia, septicemia, toxic shock syndrome(TSS), influenza A, pregnancy, autoimmune diseases, some vaccines eg measles etc ${ }^{6-10}$.

Any of these factors may trigger the onset of PRES in the patient. It has also been observed that a small percentage of patients do not have any history of hypertension. This has challenged the traditional beliefs about PRES and its etiology and has led to the speculation by some researchers that hypertension may not be the cause of the disorder but surges in the blood pressure levels could act as a trigger for the same ${ }^{9}$. Many studies believe eclampsia to be a good prognostic factor for PRES 11-15.

Although the exact hemodynamic mechanisms underlying this syndrome are not clear, three theories have been suggested ${ }^{1,2}$. The most widely accepted theory is that severe hypertension causes the auto - regulation system to fail leading to hyperperfusion and subsequent endothelial injury and vasogenic edema. The original theory suggests that hypertension or rapid changes in the blood pressure (BP) results in compensatory mechanisms of cerebral auto regulatory vasoconstriction, cerebral ischemia and resultant cerebral edema.

The most recent theory suggests that it has an immunological etiology. The immune system is triggered with T-cell activation, endothelial activation and T-cell trafficking which further stimulates vasculopathy and vasoconstriction resulting in sustained hyporperfusion and edema so very characteristic of PRES ${ }^{9-12}$.

The first theory recognizes vasospasm as the main underlying mechanism which is a resultant of overt activation of cerebral auto-regulation that might result in reversible ischemia and at the potentially risky basement membrane of the cerebral vascular tree.Most of the cases do not manifest vasospasm in the large vessels. Even if some patients do have evidence of hypo- perfusion on SPECT cerebral perfusion tests, most depict hyperperfusion findings ${ }^{17}$.

At present the hypoperfusion theory is the most widely accepted one globally. Hemostatic mechanism tries to ensure regular blood flow to the brain by auto-regulation. With the drop in systemic blood pressure, the threshold values of the auto-regulatory system dips down further accordingly. In order to increase the cerebral blood flow, the distal arterioles start to undergo vasodilatation.

Conversely, with an elevation of the systemic blood pressure, the threshold values of the auto-regulatory system rise to higher level accordingly. To increase the cerebral perfusion, the cerebral arterioles undergo vasoconstriction resulting in enhanced systemic vascular resistance.

It is a known fact that there is an upper limit for cerebral autoregulation at the time of spontaneous overt hypertension. When this limit is exceeded, the already vasoconstricted arterioles cannot undergo further contraction and then they are forced to undergo vasodilatation. This vasodilatation first initiates in the small segments sporadically and then gradually involves the whole vascular tree. This then leads to breakdown of the blood brain barrier resulting in extravasation of fluid, macromolecules along with erythrocytic extraversions which is evidenced in the brain parenchyma ${ }^{18,19}$. The cerebral cortex, owing to its compact and more organized structure, resists large cerebral edema, which then shows tendency to percolate towards the subcortical white matter by continually destroying the fragile blood brain barrier ${ }^{20}$.

After the blood brain barrier recovery has occurred, the edema is absorbed gradually from the subcortical white matter. This process is aided by the sympathetic nervous system. And because sympathetic innervation is poorer on the posterior circulation as compared to the anterior one, this results in a consequent reduced auto- 
regulation of the already impaired cerebral areas ${ }^{4,21}$. This explains the posterior brain territory susceptibility and radiological findings which are generally seen in PRES.

The alterations of cytotoxic and vasogenic edema in DWI (diffusion weighted imaging) and ADC (apparent diffusion coefficient) on imaging maps can be described as follows (Table 1):

Table 1: The alterations of cytotoxic and vasogenic edema in DWI and ADC on imaging maps

\begin{tabular}{lll}
\hline Categories & Vasogenic edema & Cytotoxic edema \\
\hline DWI & + increased signal & + increased signal \\
& intensity & intensity \\
ADC & Increased signal & Decreased signal \\
& intensity & intensity \\
Evolution & Usually reversible & Usually reversible
\end{tabular}

Biopsy or autopsy specimens obtained during an acute state depicts histological features of vasogenic edema in accordance with the observations seen on DWI. Evidences of activated or reactive astrocytes, scattered macrophages along with numerous T-Lymphocytes are seen without inflammation, ischemia or neuronal damage.

Late autopsy reports manifest evidence of demyelination and myelin pallor in addition to evidence of ischemia, neuronal anoxic damage, laminar necrosis or old hemorrhages and infarcts in the white matter and cortex $^{2,22}$.

Though the intensity and severity of the clinical manifestations of PRES may vary from case to case, nevertheless, altered mental status, confusion, headaches, seizures and symptoms related to the occipital lobe are consistently present. The symptoms may have an acute or sub-acute onset but the seizure is almost invariable present, maybe focal but usually is of generalized tonic clonic type- sometimes maybe of the status eclampticus type $2 .^{2}$

Altered mental status may manifest as anything from alertness or drowsiness and confusion to deep coma and even death eventually ${ }^{21}$. The visual manifestations may range from mild blurring of vision to cortical blindness. The patients might have normo-active deep tendon reflexes or sometimes weakness in coordination might be a elicited too ${ }^{1,7,14}$. Some patients may slow wave activity on EEG (Electro Encephalo Graphy) ${ }^{2}$.
A neuro-imaging study in PRES usually shows bilateral and symmetrical cerebral edema in the subcortical regions of the parietal and the occipital lobe in approximately $98 \%$ of the cases, corresponding to the typical manifestation of PRES which is mainly due to vasogenic edema rather than the cytotoxic variety. ${ }^{2}$ However, these lesions can also extend into the other cerebral structures e.g frontal lobes $(68 \%)$, the temporal lobes $(40 \%)$,the cerebellar hemisphere $(30 \%)$, the basal ganglia (14\%), the brainstem (13\%) and also the deep white matter, especially the splenium of the corpus callosum $(10 \%)$. In as many as $28 \%$ of the cases these involvement might be unilateral only. ${ }^{23,24}$

On CT brain, the affected areas are depicted as diffuse hypo-dense lesions, but MRI is far superior in delineating the lesions ${ }^{24}$ - which appears as iso-dense or low signal intensity on $\mathrm{T} 1$ weighted images and high signal intensity on T2 weighted images and FLAIR (Fluid Attenuated Inversion Recovery).

However FLAIR images are considered superior to proton-density and T2 weighted spin-echo for detecting supratentorial cerebral lesions. Moreover FLAIR sequences allow the neuroradiologist to have better characterization of the lesion location ${ }^{23,26,27}$.

These lesions usually resolve within days or weeks with appropriate treatment. Early diagnosis and treatment are the two main points towards achieving good prognosis with better outcomes ${ }^{29}$. The mainstay of treatment is identifying and managing the underlying triggering or precipitating factor, control and prevent seizures, strict blood pressure control and other supportive treatment as required. Correction of the systolic blood pressure is of paramount importance so as to prevent worsening of the cerebral edema ${ }^{30}$. Labetalol, hydralazine and nifidepine are quite effective in achieving the desired control over the hypertension because of their quick onset of action and easy dose titrability ${ }^{31}$.

Term and near term pregnancies must be terminated, with magnesium sulfate for eclamptic seizure prevention and control. Refractory seizures might require treatment with barbiturates e.g Phenytoin or Phenobarbital or benzodiazepinese. glorazepam ordiazepam. Patients who present with malignant PRES should be promptly referred to Neuro Critical Care Unit for neurophysical and 
neurosurgical evaluation and aggressive management of the underlying conditions ${ }^{5,31}$.

\section{Conclusion}

Posterior Reversible Encephalopathy Syndrome (PRES), despite its dramatic and overwhelming cliniconeuroradiological presentation, is potentially a reversible condition with definitive treatment. It is therefore imperative that all the clinicians should make an effort in understanding the syndrome and its etiopathogenesis so as to avoid unnecessary and unwarranted repeated neuroimaging studies. It is advocated that MRI should be used in suspected cases of PRES which can give as significant information, especially regarding the differential diagnosis of PRES and infarct of ischemic origin. DWI is especially recommended to avoid delayed diagnosis and treatment of PRES so as to ensure better prognosis.

\section{Conflict of interest: None. Disclaimer: Nil.}

\section{References}

1.Hinchey J, Chaves C, Appignani B, Breen J, Pao L, Wang A, Pessin MS, et al. A reversible posterior leukoencephalopathy syndrome. N Eng J Med. 1996; 334: 494-500.

2.Buyukaslan H, Lok U, Gulacti U, Sogut O, Kaya H, Gokdemir T, et al. Case report of 4 PRES in peripartum period. Int J clin Exp Med. 2015; 8(2):1575-81.

3.Oyinloye OI, Adesiyun O, Atobatele MO, Fawole AA. PRES in an adult female. Ann Afr Med. 2014; 13: 138-41.

4.Hegde HV, Rao RP. PRES in a patient of eclampsia with partial HELLP Syndrome. Current Anesthesia Critical Care. 2009; 20(2): 251-53.

5.Akins PT, Axelrod Y, Silverthorn JW, Guppy K. Management \& Outcomes of PRES Clin Neurol neurosurg. 2014;125: 52-57.

6.Doherty H, Hameed S, Ahmed I, Russell IF. Postdural puncture headache \& PRES. Int J Obstet Anesth. 2014; 23 : 279-82.

7.Brewer J, Owens MY, Wallace K, Reeves AA, Morris R, Khan M. PRES in 46 of 47 patients with eclampsia. Am J Obstet Gynecol. 2013; 208(6): 468.e1-6.

8.Ringelstein EB. Cerebral small vessel diseases: manifestations in young women. Current opinion Neurology. 2006; 19: 55-62.
9.Arimura FE, Camargo PC, Costa AN, Teixeira RH, Carraro RM, Afonso. PRES in lung transplantation: 5 case reports. Transplant Proc. 2014; 46: 1845-48.

10.Ay H, Buonanno FS, Schaefer PW, Le DA, Wang B, Gonzalez R, et al. PRES without severe hypertension. Neurology. 1998; 51: 1369-76.

11.Roy S, Gandhi AK, Jana M, Julka PK. Recurrent PRES after chemotherapy in hematologic malignancy. J cancer Res Thu. 2014; 10: 393-96.

12.Hamano T, Takeda T, Morita H, Muramatsu T, Yoneda M, Kimura H, Kuriyama M. PRES following measles vaccination J Neurol Sci. 2010; 298: 124-26.

13. Yano Y, Kario K, Fukunaga T, Ohshita T, Himeji D, Yano M, Nakagawa S, Sakata Y, Shimada K. A case reversible posterior leukoensephalopathy syndrome caused by transient hypercoagulable state induced by infection. Hypertens Res. 2005; 28: 619-23.

14.Pande AR, Ando K, Ishikura R, Nagami Y, Takada Y, Wada A, Watanabe Y, Miki Y, Uchino A, Nakao N. Clinicoradiological factors influencing the reversibility of posterior reversible encephalopathy syndrome: a multicenter study. Radiat Med. 2006; 24: 659-68.

15.Fitzgerald RT, Wright SM, Samant RS, Kumar M, Ramakrishnaiah RH, Van Hemert R, Brown AT, Angtuaco EJ. Elevation of serum lactate dehydrogenase at posterior reversible encephalopathy syndrome onset in chemotherapytreated cancer patients. J Clin Neurosci. 2014; 21: 1575-78.

16.Rabinstein AA, Mandrekar J, Merrell R, Kozak OS, Durosaro O, Fuqate JE. Blood pressure fluctuations in posterior reversible encephalopathy syndrome. J Stroke Cerebrovasc Dis. 2012; 21: 254-8.

17.Szer IS, Miller JH, Rawlings D, Shaham B, Bernstein B. Cerebral perfusion abnormalities in children with central nervous system manifastations of lupus detected by single photon emission computed tomography. J Rheumatol. 1993; 20: $2143-48$.

18.Mackenzie ET, Strandgaard S, Graham DI, Jones JV, Harper AM, Farrar JK. Effects of acutely induced hypertension in cats on pial arteriolar caliber, local cerebral flow, and the blood-barrier. Circ Res. 1976; 39: 33-41.

19.Grossbach AJ, Abel TJ, Hodis B, Wassef SN, Greenlee JD. Hypertensive posterior reversible encephalopathy syndrome causing posterior fossa edema and hydrocephalus. J Clin Neurosci. 2014;21:207-211.

20.Kalimo H, Fredriksson K, Norborg C, Auer RN, Olsson $\mathrm{Y}$, Johansson B. The spread of brain oedema in hypertensive brain injury. Med Biol. 1986; 64: 133-37. 
21.Edvinsson L, Owman C, Sjoberg NO. Autonomic nerves, mast cells, and amine receptors in human brain vessels. A histochemical and pharmacological study. Brain Res.

1976;115:377-393.

22.Schiff D, Lopes MB. Neuropathological correlates of reversible posterior leukoencephalopathy. Neurocrit Care. 2005; 2: 303-05.

23.Hugonneta E, Inesa DD, Boby H, Claise B, Petitcolin V, Lannareix V, Garcier JM. Posterior reversible encephalopathy syndrome (PRES): features on CT and MR imaging. Diagn Interv Imaging. 2013;94:45-52.

24.Wu Q, Marescaux C, Qin X, Kessler R, Yang J. Heterogeneity of radiological spectrum in tacrolimusassociated encephalopathy after lung transplantation. Behav Neurol. 2014;2014:931808.

25.Patil VS, Dhaman gaon kar BR, Pattanshetti RC, Patil MM. Posterior reversible encephalopathy syndrome in early postpartum women: a case report. J Clin Diagn Res. 2014; 8: RD01-RD02.

26.McKinney AM, Short J, Truwit CL, McKinney ZJ, Kozak OS, SantaCruz KS, Teksam M. Posterior reversible encephalopathy syndrome: incidence of atypical regions of involvement and imaging findings. AJR Am J Roentgenol. 2007;189:904-12.
27.Casey SO, Sampaio RC, Michel E, Truwit CL. Posterior reversible encephalopathy syndrome: utility of fluidattenuated inversion recovery MR imaging in the detection of cortical and subcortical lesions. Am J Neuroradiol.2000;21:1199-06.

28.Leutmer PH, et al. PRES in children. Pediatr Emerg Care. 2012; 28:153-57.

29.Endo A, Fuchigami T, Hasegawa M, Hashimoto K, Fujita Y, Inamo Y, Mugishima H. Posterior reversible encephalopathy syndrome in childhood report of four cases and review of the literature. Pediatr Emerg Care.2012;28:153-7.

30.Schusse CM, Peterson AL, Caplan JP. Posterior reversible encephalopathy syndrome. Psychosomatics.2013;54:205-11.

31.Lang CR, Coeller N. Posterior reversible encephalopathy syndrome: a unique presentation. Am J Emerg

Med.2013;31:1423, e3-4.

\section{Jayati Nath ${ }^{1}$}

${ }^{1}$ Professor, Obstetrics and Gynecology, MMIMSR, Mullana, Ambala, Haryana. 